\title{
Density of Liquid Tantalum and Estimation of Critical Point Data
}

\author{
M. Leitner ${ }^{1} \mathbb{D} \cdot$ W. Schröer ${ }^{2} \cdot$ G. Pottlacher ${ }^{1}$
}

Received: 16 October 2017 / Accepted: 13 August 2018 / Published online: 3 September 2018

(c) The Author(s) 2018

\begin{abstract}
In order to determine the density of tantalum over the entire liquid phase (at the pressure applied) and several hundred $\mathrm{K}$ into the super-heated region, the method of ohmic pulse-heating was applied. For this purpose, images of the thermal radial expansion of the resistively heated sample wires were taken with an adapted CCD system. A newly integrated high-power photoflash and improved triggering of the experiment allowed the acquisition of high-contrast shadow images of the expanding wires. To reduce the uncertainty arising from simultaneous pyrometric temperature measurement, the change in normal spectral emissivity as a function of temperature was additionally taken into account. In this work, the density versus temperature relationship of tantalum is reported and compared to existing literature data. From the newly obtained liquidphase density, critical point data of tantalum, such as critical temperature and critical density, were estimated via an extrapolation procedure. Furthermore, an estimate of the phase diagram in the density versus temperature plane is given. The work is concluded by a rigorous density uncertainty estimation according to the guide to the expression of uncertainty in measurement (GUM).
\end{abstract}

Keywords Critical point data $\cdot$ Liquid-phase density $\cdot$ Phase diagram · Pulse-heating · Tantalum

$\bowtie \quad$ M. Leitner

matthias.leitner@tugraz.at

W. Schröer

schroer@uni-bremen.de

G. Pottlacher

pottlacher@tugraz.at

1 Institute of Experimental Physics, Graz University of Technology, NAWI Graz, Petersgasse 16, 8010 Graz, Austria

2 Faculty 02: Biology/Chemistry, Universität Bremen, Leobener Str. NW 2, 28334 Bremen, Germany 


\section{Introduction}

Critical point data for high-melting metals are scarce but of fundamental interest. Due to the extremely high temperature and pressure at this point, it cannot easily be reached experimentally for high-melting metals. However, extrapolating the liquidphase density according to theoretical models allows researchers to estimate the critical point [1]. Even though density data for high-melting metals exist in literature, they often suffer from large uncertainties or are not consistent with each other. The situation is aggravated by the fact that the data often do not reach far beyond the melting point.

In order to obtain the best starting conditions for the extrapolation procedure, two aspects are crucial. First, density data should extend as far as possible into the liquid phase, and second, the data should exhibit smallest possible uncertainties. To meet both requirements, the density of liquid tantalum was re-measured in this work by means of ohmic pulse-heating.

For recent density data of this group, please refer to [2-4]. For previous critical point data estimation on low-melting metals, please refer to, e.g., $[5,6]$.

\section{Experimental Procedure and Data Evaluation}

Tantalum wires with a diameter of $0.5 \mathrm{~mm}$ and a length of $40 \mathrm{~mm}$ (Co. Advent, purity: 99.9 wt\%, catalogue no.: Ta550615, charge no.: Gi1109, temper annealed) were resistively heated under $\mathrm{N}_{2}$ atmosphere $(2.3$ bar) by means of ohmic pulseheating, starting at room temperature $(293 \mathrm{~K})$. The slight overpressure that ensues in the sample chamber inhibits flash arcs between sharp edges of the sample-holder. Before each experiment, the samples were treated with abrasive paper (grade 1200) and subsequently cleaned with acetone.

The energy for the experiment is provided by a $500 \mu \mathrm{F}$ capacitor bank that can be charged up to $10 \mathrm{kV}$. Upon triggering the experiment, a current pulse peaking at about $10 \mathrm{kA}$ is discharged over the sample. Due to its ohmic resistance, the wire is heated from room temperature to the liquid phase until it explodes due to the sudden increase in volume at the liquid-gas-phase boundary. Heating rates of the order $10^{8} \mathrm{~K} \cdot \mathrm{s}^{-1}$ are reached. As a consequence of the high heating rates applied, the experimental duration is very short, in this case $43 \mu \mathrm{s}$. Measurements can thus be performed on the expanding liquid metal column that, due to its inertia, is vertically standing during the experiment. Besides, radiative losses, chemical interactions and evaporation effects are largely inhibited due to the short timescale. Still, the sample remains in thermodynamic equilibrium (private communication Prof. G. Pottlacher with Prof. F. Hensel, Univ. Marburg, Germany, 1986) . For a more in-depth description of the pulse-heating setup, please refer to previous publications from this working group, e.g., [3,7,8].

Investigations in the past have also indicated that high heating rates result in an increased radial wire expansion while inhibiting longitudinal expansion $[9,10]$. Ohmic pulse-heating can thus be applied to deduce the material's temperature-dependent density by monitoring the thermal radial expansion and, at the same time, record the temperature. In total, data of seven independent experiments were evaluated. 
Fig. 1 Radiance-over-time development in a pulse-heating experiment on tantalum. To calculate the calibration constant of the pyrometer, the signal at the melting plateau $J\left(T_{\mathrm{r}, \mathrm{m}}\right)$ is assigned to the radiance temperature at melting $T_{\mathrm{r}, \mathrm{m}}$

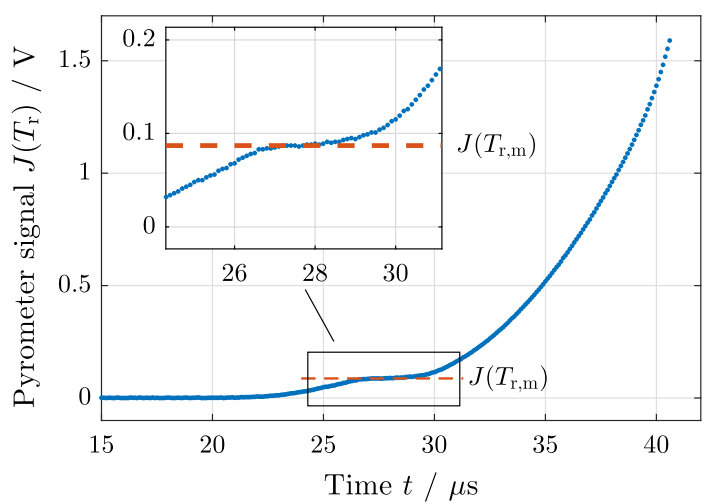

\subsection{Temperature}

In order to deduce the sample temperature, the surface radiance of the sample is monitored pyrometrically as a function of time (sampling rate: $10 \mathrm{MHz}$ ). The pyrometer used operates at a mean effective wavelength of $\lambda=652 \mathrm{~nm}$ with a filter full-widthat-half-maximum of $27 \mathrm{~nm}$.

In order to convert the recorded pyrometer voltage signal to a temperature, each experiment is self-calibrated at the melting plateau, which can be observed in the radiance-over-time development (see Fig. 1). Following Planck's law of radiation, the calibration constant $K$ can be calculated from

$$
K=J\left(T_{\mathrm{r}, \mathrm{m}}\right) \cdot\left\{\exp \left(\frac{c_{2}}{\lambda \cdot T_{\mathrm{r}, \mathrm{m}}}\right)-1\right\}
$$

where $T_{\mathrm{r}, \mathrm{m}}$ is the radiance temperature at melting, $J\left(T_{\mathrm{r}, \mathrm{m}}\right)$ is the pyrometer signal at the melting plateau, and $c_{2}=14.388 \mu \mathrm{m} \mathrm{K}$ is the second radiation constant. The radiance temperature at the melting point is calculated from the literature value for the true melting temperature $\left(T_{\mathrm{m}}=3280 \mathrm{~K}\right.$ [11]) and the normal spectral emissivity of tantalum at the melting point that was reported by Cagran et al. $\left(\varepsilon_{\mathrm{m}}=0.366\right)$ [12].

Knowing the calibration constant $K$, the pyrometer signal $J\left(T_{\mathrm{r}}\right)$ is converted into a radiance temperature $T_{\mathrm{r}}$ with

$$
T_{\mathrm{r}}=\frac{c_{2}}{\lambda \cdot \ln \left(\frac{K}{J\left(T_{\mathrm{r}}\right)}+1\right)} .
$$

Finally, the true temperature $T$ above melting is deduced from the radiance temperature $T_{\mathrm{r}}$, and the liquid-phase normal spectral emissivity $\varepsilon\left(\lambda, T_{\mathrm{r}}\right)$, according to

$$
T=\frac{c_{2}}{\lambda \cdot \ln \left\{\varepsilon\left(\lambda, T_{\mathrm{r}}\right) \cdot\left[\exp \left(\frac{c_{2}}{\lambda \cdot T_{\mathrm{r}}}\right)-1\right]+1\right\}},
$$




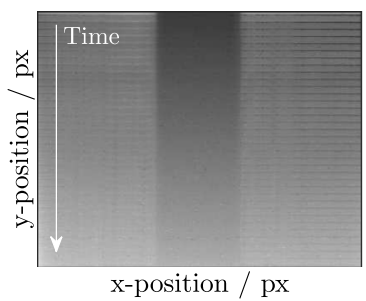

(a)

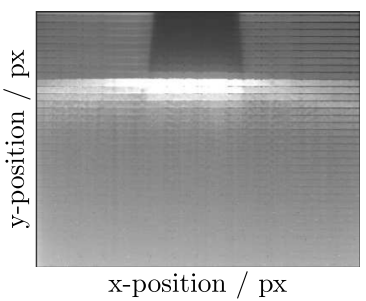

(b)

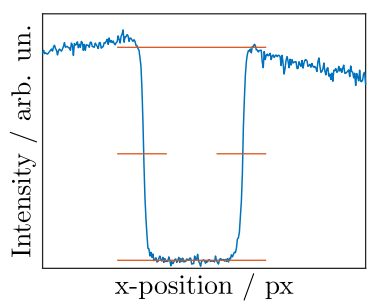

(c)

Fig. 2 (a) Image sequence captured prior to the pulse-heating start. (b) Image sequence taken during the pulse-heating experiment. Radial expansion of the wire and the phase explosion can be observed (bright horizontal band). Time and temperature can be assigned to each of the image slices. (c) Exemplary intensity profile of one streak image to deduce a precise diameter (FWHM) for density determination. These profiles are calculated by summation over the lines of the respective images

with the normal spectral emissivity given as

$$
\varepsilon\left(684.5 \mathrm{~nm}, T_{\mathrm{r}}\right)=0.49725-4.63794 \times 10^{-5} \cdot T_{\mathrm{r}},
$$

which is valid in the range $2820 \mathrm{~K}<T_{\mathrm{r}}<4400 \mathrm{~K}$ [12].

Note that the normal spectral emissivity was measured at a wavelength of $684.5 \mathrm{~nm}$, whereas the pyrometer operates at a mean effective wavelength of $652 \mathrm{~nm}$. However, due to the feasibility of a gray-body assumption in this narrow wavelength interval, the cited emissivity can be applied to Eq. 3 as stated in (4). It is important to mention that in the given experiment, an assumption of a temperature-independent liquid-phase emissivity for temperature deduction would result in an error of more than $-400{ }^{\circ} \mathrm{C}$ at the highest temperatures measured.

Due to the strong surface treatment dependence of the emittance in the solid phase, see, e.g., [13], it is difficult to find valuable reference data. The temperature in the solid phase was thus calculated under the assumption that the emittance is independent of temperature and takes the value that is true at the melting point.

\subsection{Thermal Radial Expansion and Density}

The thermal radial expansion of the wire is monitored by an adapted CCD system that is mounted orthogonally to the direction of the pyrometer view. The expanding wire is backlit by the collimated light of a newly integrated high-power photoflash (Multiblitz X10, $1000 \mathrm{Ws}$ ). The shadowgraph is imaged with the adapted CCD system that first converts the incident photons into electrons (photocathode), then amplifies the signal (multi-channel-plate) and finally reconverts the electrons into photons (phosphor screen) for subsequent imaging onto a CCD chip (sensor, $384 \times 572$ pixel). The sensor is mechanically masked such that only 8 pixel lines are uncovered and thus ready for exposure. The rest of the chip serves as a fast buffer storage unit for the recorded streak images that are shifted into the masked region after acquisition. This way, numerous images can be recorded for each experiment (see Fig. 2a, b), because the time-expensive reading of the chip information can be postponed. 
Time synchronization is provided by a common trigger pulse such that all measured quantities share the same time basis. A point in time and, therefore, a temperature can thus be assigned to each of the recorded image slices. Due to the high intensity of the photoflash, the exposure time could be decreased to $300 \mathrm{~ns}$. The setup now takes images about every $2.5 \mu \mathrm{s}$, which corresponds to a frame rate of $4 \times 10^{5} \mathrm{fps}$. For more technical details about the CCD system, see, e.g., [10,14].

Figure 2 depicts a typical image sequence that is acquired before the pulse-heating experiment (a) and a sequence that is taken during the experiment (b). Note that the time propagates from top to bottom. By evaluating the full-width-at-half-maximum of the calculated intensity profiles (Fig. 2c) of each streak image, the volume expansion $V(T) / V_{0} \equiv\left(d(T) / d_{0}\right)^{2}$ and from that the density $\rho(T)$ can be calculated via

$$
\rho(T)=\rho_{0} \cdot\left(\frac{d_{0}}{d(T)}\right)^{2}
$$

where $\rho_{0}$ is the room temperature density and $d_{0}$ and $d(T)$ are the evaluated diameters obtained from the image sequence. Due to the experimental constraints, the quadratic radial expansion represents the volume expansion as indicated above.

Note that the contrast of the streak images decreases as a function of time (see Fig. 2a). As it takes about $20 \mu$ s counted from the initiation of pulse-heating, until the sample's surface radiance is high enough to be detected by the pyrometer, the camera start is timed accordingly to obtain high-contrast streak images with steep edges in the intensity profiles.

To obtain a homogeneous distribution of expansion values with respect to temperature, the camera start is shifted by steps of $0.5 \mu$ s between consecutive experiments.

\subsection{Critical Point Data}

The estimation of critical point data such as critical temperature $T_{\mathrm{c}}$ and critical density $\rho_{\mathrm{c}}$ is based on an extrapolation of the liquid-phase density $\rho(T)$, as proposed by Schröer and Pottlacher [1].

The main idea is to fit the liquid-phase density, indexed by a plus, according to Ising behavior $\rho_{+} \propto\left(T_{\mathrm{c}, \mathrm{I}}-T\right)^{1 / 3}$ and mean field behavior $\rho_{+} \propto\left(T_{\mathrm{c}, \mathrm{mf}}-T\right)^{1 / 2}$, yielding estimates for the critical temperature (fit coefficients) according to the model used. A final estimate for the critical temperature $T_{\mathrm{c}}$ is obtained by taking the arithmetic mean between the two values $T_{\mathrm{c}, \mathrm{I}}$ and $T_{\mathrm{c}, \mathrm{mf}}$.

Once this value has been obtained, estimates for the critical density $\rho_{\mathrm{c}}$ are determined in two ways. First, the rule of rectilinear diameter is applied, meaning that $\rho_{\text {diam }}=\rho_{\mathrm{c} \text {, lin }}+a_{\text {lin }} \cdot\left(T_{\mathrm{c}}-T\right)$ is fitted to the diameter $\frac{\left(\rho_{+}+\rho_{-}\right)}{2}$, which can be approximated by $\rho_{\text {diam }}=\frac{\rho_{+}}{2}$ in the measuring region (compare Fig. 4 ). One of the two fitting parameters yields the critical density $\rho_{\mathrm{c}}$,lin. In the second approach, the phase diagram diameter is fitted according to $\rho_{\text {diam }}=\rho_{\mathrm{c}, 2 / 3}+a_{2 / 3} \cdot\left(T_{\mathrm{c}}-T\right)^{2 / 3}$, again giving an estimate for the critical density, i.e., $\rho_{\mathrm{c}, 2 / 3}$. The average of these two critical densities is taken as final estimate for $\rho_{\mathrm{c}}$. 
The phase diagram is then obtained by fitting the diameter $\rho_{\text {diam }}$ according to

$$
\rho_{\text {diam }}=\frac{\rho_{+}}{2}=\rho_{\mathrm{c}}\left(1+a \cdot\left(T_{\mathrm{c}}-T\right)+c \cdot\left(T_{\mathrm{c}}-T\right)^{2 / 3}\right),
$$

yielding the fitting parameters $a$ and $c$. The liquid-phase density $\rho_{+}$is then fitted following Eq. 7,

$$
\rho_{ \pm}=\rho_{\text {diam }} \pm b \cdot\left(T_{\mathrm{c}}-T\right)^{1 / 3}\left(1+b_{2} \cdot\left(T_{\mathrm{c}}-T\right)^{2 / 3}\right)
$$

and the parameters $b$ and $b_{2}$ are obtained. $\rho_{-}$describes the gas phase density.

For Alkali metals, estimates for critical data $T_{\mathrm{c}}$ and $\rho_{\mathrm{c}}$, obtained by taking the arithmetic mean between the extrapolated values presuming Ising or mean field behavior, were in good agreement with experimentally determined critical point data [1]. Thus, this approach was applied here too.

\section{Results and Discussion}

In this section, results are depicted in graphical form and compared to those appearing in the existing literature. Relevant details about the references used for density comparison are listed in Table 1. Note the differences in experimental pressure applied. However, due to the low isothermal compressibility of liquid Ta, $\kappa_{\mathrm{T}}=0.0168 \mathrm{GPa}^{-1}$ (at the melting point) [15], pressure-related deviations in density should be less than $0.5 \%$. The experimentally obtained liquid-phase density values are listed in Table 3 . The regression to the data is given in the form of a polynomial (Table 2).

\subsection{Density}

To derive density data from the measured thermal radial expansion, a room temperature density of $\rho_{0}=16654 \mathrm{~kg} \cdot \mathrm{m}^{-3}$ was adopted from [20]. The result is depicted in Fig. 3 together with data given in the literature and shows a linear decrease with increasing temperature. A linear regression to the density data in the liquid phase was made. At the beginning of the liquid phase, a density of $\rho\left(T_{\mathrm{m}, 1}\right)=(15.01 \pm 0.21) \times 10^{3} \mathrm{~kg} \cdot \mathrm{m}^{-3}$ is obtained by evaluating the fit equation given in Table 2. Based on the boiling temperature of tantalum $T_{\mathrm{b}}=5731 \mathrm{~K}$ [20], a super-heating of about $670 \mathrm{~K}$ is achieved before the wire explodes (Table 3).

The data reported by Gathers [16], Berthault et al. [17] and Jäger et al. [18] were originally given as volume expansions $V(T) / V_{0}$. In the first two articles, either the specific volume $V_{0}$ or the room temperature density was also stated and the density could thus be calculated straightaway. For the data of Jäger et al., the $\rho_{0}$ value given above was applied to convert the volume expansion into density.

Paradis et al. [19] measured the density of tantalum in the liquid and supercooled liquid state via an electrostatic levitation technique. The comparison between Paradis's data and the data of this work is thus of particular interest due to the completely different methods applied. 


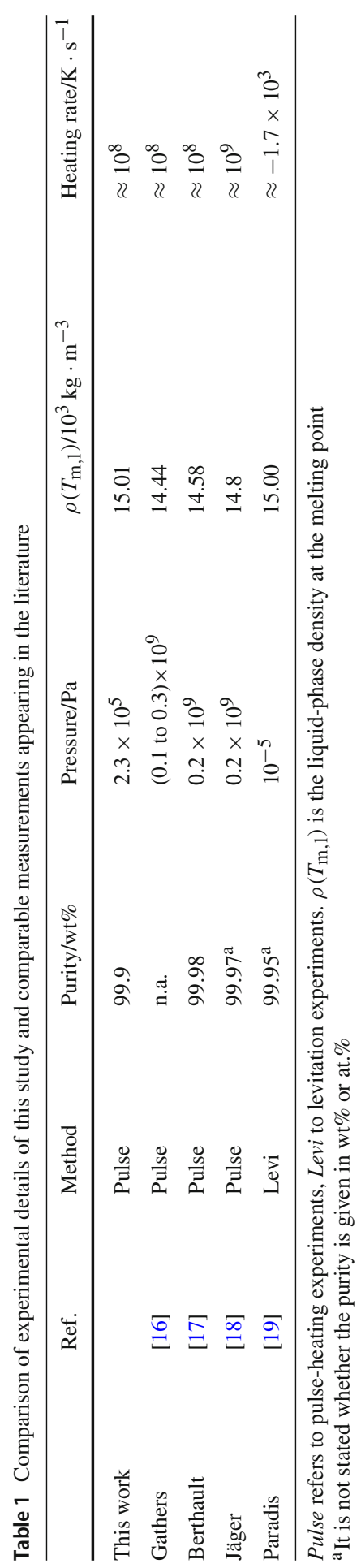


Table 2 Fit coefficients for the liquid-phase density of tantalum in the form $\rho(T)=a-b \cdot T$

\begin{tabular}{llll}
\hline Equation & $a / \mathrm{kg} \cdot \mathrm{m}^{-3}$ & $b / \mathrm{kg} \cdot \mathrm{m}^{-3} \cdot \mathrm{K}^{-1}$ & Temperature range \\
\hline$\rho(T)=a-b \cdot T$ & $(17.25 \pm 0.17) \times 10^{3}$ & $(0.684 \pm 0.034)$ & $3280 \leq T / \mathrm{K} \leq 6400$
\end{tabular}

The density uncertainty calculated with the given fit increases from $1.4 \%$ at the beginning of the liquid phase to $2.3 \%$ at the highest temperature. Uncertainties are given with $k=2$. Note that an additional, insignificant digit is given for the slope $b$ in order to retain the original fitting curve. This curve is used in a second step to estimate critical point data

Table 3 Experimental values of density as a function of temperature $\rho(T)$ for tantalum derived from thermal expansion measurements

\begin{tabular}{llllll}
\hline$T / \mathrm{K}$ & $\rho(T) / \mathrm{kg} \cdot \mathrm{m}^{-3}$ & $T / \mathrm{K}$ & $\rho(T) / \mathrm{kg} \cdot \mathrm{m}^{-3}$ & $T / \mathrm{K}$ & $\rho(T) / \mathrm{kg} \cdot \mathrm{m}^{-3}$ \\
\hline 2084 & 16131 & 3299 & 14941 & 4715 & 14370 \\
2102 & 16128 & 3303 & 14972 & 4765 & 13716 \\
2145 & 16094 & 3304 & 15132 & 4855 & 13837 \\
2263 & 15924 & 3343 & 15010 & 4877 & 14029 \\
2455 & 15731 & 3361 & 15028 & 4978 & 14071 \\
2616 & 15891 & 3467 & 14850 & 5090 & 13710 \\
2670 & 15566 & 3586 & 14805 & 5286 & 13624 \\
2672 & 15776 & 3631 & 14520 & 5289 & 13894 \\
2698 & 15675 & 3653 & 14743 & 5406 & 13248 \\
2855 & 15659 & 3663 & 14601 & 5490 & 13352 \\
2912 & 15670 & 3804 & 14666 & 5521 & 13598 \\
3035 & 15495 & 3864 & 14420 & 5589 & 13473 \\
3127 & 15512 & 4045 & 14509 & 5734 & 13286 \\
3166 & 15273 & 4143 & 14495 & 5856 & 13149 \\
3168 & 15416 & 4174 & 14221 & 5938 & 13459 \\
3175 & 15326 & 4244 & 14241 & 6095 & 12932 \\
3259 & 15344 & 4252 & 14351 & 6186 & 13051 \\
3273 & 15424 & 4382 & 14427 & 6217 & 12907 \\
3281 & 15274 & 4466 & 14108 & 6349 & 12755 \\
3290 & 15111 & 4658 & 14341 & 6400 & 12944 \\
\hline & 152 & & & 569 \\
\hline
\end{tabular}

The density values were obtained with a room temperature density of $\rho_{0}=16654 \mathrm{~kg} \cdot \mathrm{m}^{-3}$. Combined expanded temperature uncertainty for the liquid phase $(T>3280 \mathrm{~K}), U_{\mathrm{c}}(T) / T=0.017$ at the beginning of the liquid phase up to $U_{\mathrm{c}}(T) / T=0.028$ at the highest temperature measured. Combined expanded density uncertainty $U_{\mathrm{c}}(\rho) / \rho=0.013$. Uncertainties reported with a level of confidence $0.95(k=2)$

As can be seen in Fig. 3, the newly measured density data are in excellent agreement with the data found by Paradis et al. At the melting point, Paradis et al. reported $\rho\left(T_{\mathrm{m}}\right)=15.0 \times 10^{3} \mathrm{~kg} \cdot \mathrm{m}^{-3}$ which corresponds to a deviation of only $-0.07 \%$ with respect to the value derived in this work. The uncertainty is stated with $<2 \%$. To deduce the temperature, they assumed a constant emissivity behavior in the liquid phase. Also note the difference of ten orders of magnitude in the experimental pressure 


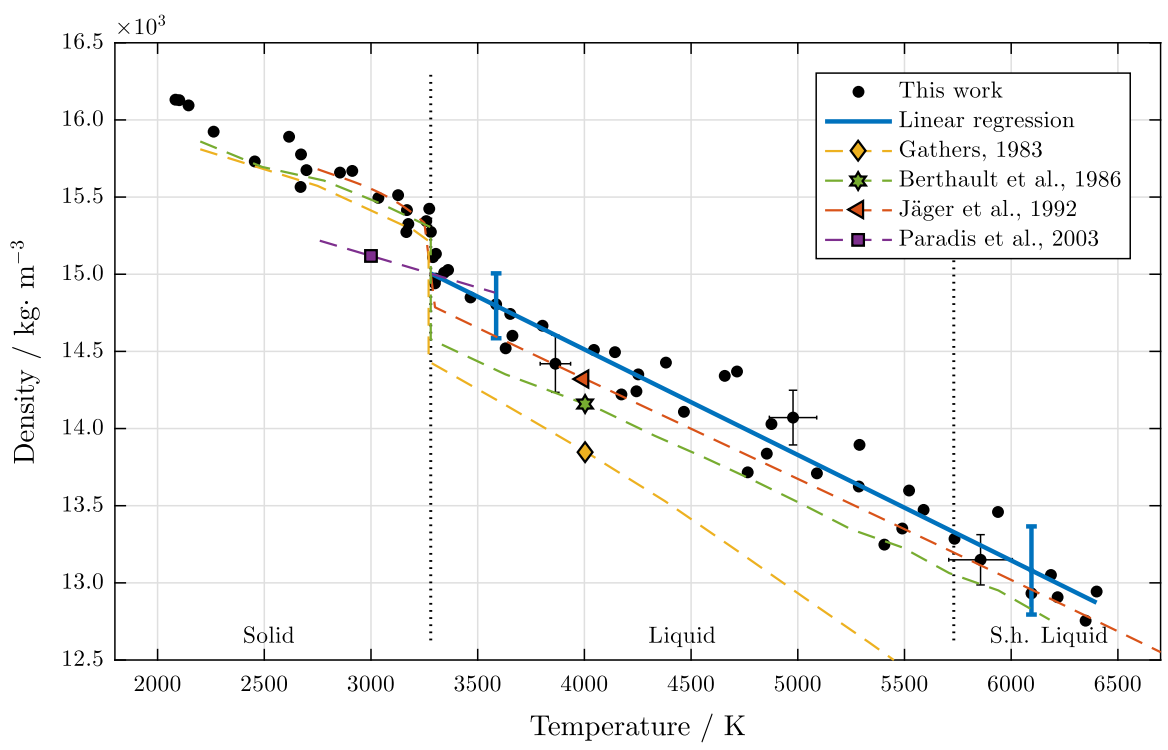

Fig. 3 Density of tantalum as a function of temperature. The vertical dotted lines delineate the melting and boiling temperatures. Above the boiling point, the sample is in a super-heated (S.h.) liquid state. Full circles and solid line: experimental data obtained during this work and corresponding liquid-phase linear regression. Gathers [16]: dashed line with diamond, Berthault et al. [17]: dashed line with star, Jäger et al. [18]: dashed line with triangle, Paradis et al. [19]: dashed line with square, measurements in the liquid and undercooled liquid state

applied and the difference between a heating rate of $10^{8} \mathrm{~K} \cdot \mathrm{s}^{-1}$ in our experiments as compared to a cooling rate of about $-1.7 \times 10^{3} \mathrm{~K} \cdot \mathrm{s}^{-1}$ achieved by Paradis et al.

The data obtained in the present study are also in very close agreement with the data reported earlier by our group (Jäger et al. [18]) as well as the data published by Berthault et al. From the data of Jäger et al., a density at the beginning of melting of $\rho\left(T_{\mathrm{m}, 1}\right)=14.8 \times 10^{3} \mathrm{~kg} \cdot \mathrm{m}^{-3}$ can be derived. This corresponds to a deviation of only $-1.4 \%$ with respect to our data. This is remarkable, since they used different wire diameters $(0.25 \mathrm{~mm})$, a heating rate that is higher by one order of magnitude (about $10^{9} \mathrm{~K} \cdot \mathrm{s}^{-1}$ ), and took photographs of the expanding wire with a Kerr-cell camera, which can only take one picture per experiment (30 ns exposure time). Also note that these experiments were performed at a pressure that is three orders of magnitude higher than in our experiments. A constant emissivity in the liquid phase was assumed in the temperature deduction. Jäger et al. state an uncertainty of $8 \%$ for volume expansion. However, as the coverage factor is not reported in the original publication, it is not converted into a density uncertainty here. The same is true for the data given by Berthault et al.; they state an uncertainty of $2 \%$ for volume expansion. The data given by Berthault et al. are even lower, $\rho\left(T_{\mathrm{m}, 1}\right)=14.58 \times 10^{3} \mathrm{~kg} \cdot \mathrm{m}^{-3}(-2.9 \%)$, but still in reasonable agreement with our data. It was also assumed that the emissivity does not change in the liquid phase. These experiments were performed under a pressure of $0.2 \mathrm{GPa}$. 


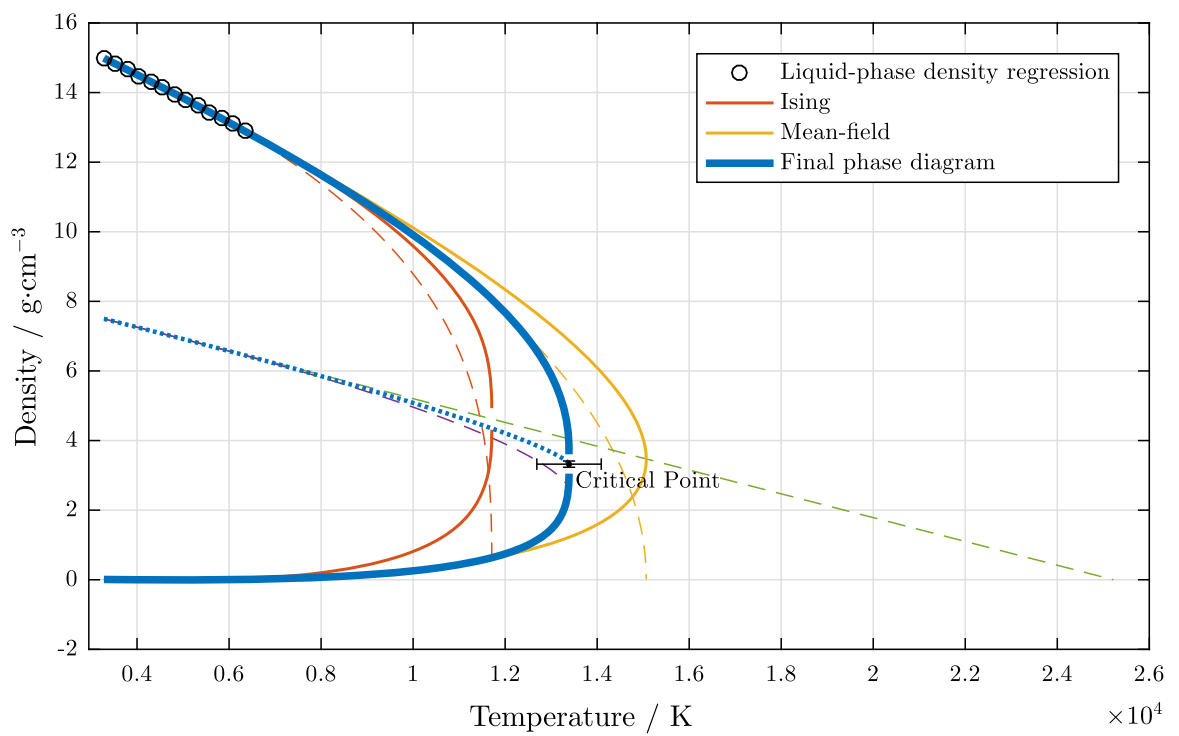

Fig. 4 Estimated phase diagram (thick solid line) with nonlinear diameter $\rho_{\text {diam }}$ (dotted line) and critical point of tantalum. Open circles: Data generated by the linear regression of this work's experimental density data. Dashed lines: Extrapolation of the density data (fifty data points) according to mean field and Ising behavior as well as linear and nonlinear extrapolation of the phase diagram diameter. Thin solid lines: Phase diagrams according to mean field and Ising behavior

The high-pressure data measured by Shaner et al. [21] and later corrected by Gathers are about $3.8 \%$ lower at the beginning of the liquid phase $\left(\rho\left(T_{\mathrm{m}, 1}\right)=\right.$ $\left.14.44 \times 10^{3} \mathrm{~kg} \cdot \mathrm{m}^{-3}\right)$. The discrepancy further increases at higher temperatures due to the difference in slope. Gathers estimated the emissivity from the pyrometer signal via a numerical procedure. No uncertainty is given.

\subsection{Critical Point Data}

The critical point in the $(\rho, T)$-plane was estimated according to the proposal of Schröer and Pottlacher [1] as briefly outlined in Sect. 2.3. Figure 4 shows density data points far away from the critical point that are used for the extrapolation (data calculated from the linear regression, Table 2). Fifty data points are fitted according to mean field and Ising theory (dashed lines). The straight dashed line shows the phase diagram diameter fit according to the rule of rectilinear diameter. Once these data have been obtained, the phase diagram according to the Ising model and to mean field theory can be plotted (thin solid lines). As outlined in Sect. 4.2, the phase diagram diameter is also fitted in a nonlinear manner (curved dashed line).

The final estimate of the phase diagram is depicted as a thick solid line, described by Eq. 7 . The dotted line shows the nonlinear diameter of the estimated phase diagram according to Eq. 6. The fit coefficients needed to describe the phase diagram are listed in Table 4. Uncertainty bars show the range of variation of the critical point due to the uncertainty of the density-fit parameters, see Sect. 4.2. 
Table 4 Parameters of the estimated phase diagram of tantalum according to Eqs. 6 and 7

\begin{tabular}{llll}
\hline$a / 10^{-5} \mathrm{~K}^{-1}$ & $c / 10^{-3} \mathrm{~K}^{-2 / 3}$ & $b / 10^{-1} \mathrm{~K}^{-1 / 3}$ & $b_{2} / 10^{-4} \mathrm{~K}^{-2 / 3}$ \\
\hline 5.16 & 1.57 & 2.98 & 3.48 \\
\hline
\end{tabular}

For the critical temperature $T_{\mathrm{c}}$ and the critical density $\rho_{\mathrm{c}}$, we obtain

$$
\begin{aligned}
& T_{\mathrm{c}}=(13.4 \pm 0.7) \times 10^{3} \mathrm{~K} \\
& \rho_{\mathrm{c}}=(3.32 \pm 0.09) \mathrm{g} \cdot \mathrm{cm}^{-3}
\end{aligned}
$$

Critical temperatures estimated in the literature range from $T_{\mathrm{c}}=8865 \mathrm{~K}$ [22] to values as high as $T_{\mathrm{c}}=22000 \mathrm{~K}$ [23] depending on the method and input data used. For a detailed listing of several theoretically predicted $T_{\mathrm{c}}$ values, we would like to refer the reader to the publication of Blairs and Abbasi [24] and the references therein.

A rather recent work by Blairs and Abbasi [25] should also be mentioned in this context. Critical temperatures for metals, obtained via two different methods, are reported by these authors and compared to selected literature values. For tantalum, they obtain $T_{\mathrm{c}}=13284 \mathrm{~K}$ and $T_{\mathrm{c}}=14238 \mathrm{~K}$ in their study. Both of these values are in excellent agreement with the result of this study.

Fortov et al. [26] report $T_{\mathrm{c}}=13380 \mathrm{~K}, \rho_{\mathrm{c}}=3.83 \mathrm{~g} \cdot \mathrm{cm}^{-3}$, which is in very good agreement with our data. In addition, they report a critical pressure of $p_{\mathrm{c}}=0.707 \mathrm{GPa}$. In their publication, a phase diagram can be found together with other $\left(T_{\mathrm{c}}, \rho_{\mathrm{c}}\right)$-values. In general, a downward trend of the reported critical temperatures can be observed over the years. Older reported values peak between $20000 \mathrm{~K}$ and $22000 \mathrm{~K}$, while more recent values peak in the range between $12000 \mathrm{~K}$ and $14000 \mathrm{~K}$. For the critical density, values reported in the literature range between $\rho_{\mathrm{c}}=1.9 \mathrm{~g} \cdot \mathrm{cm}^{-3}$ [22] and $\rho_{\mathrm{c}}=6.72 \mathrm{~g} \cdot \mathrm{cm}^{-3}[27]$.

\section{Uncertainties}

This section deals with uncertainty estimation according to the Guide to the expression of uncertainty in measurement, shortly referred to as GUM [28].

\subsection{Density}

The data point error bars depicted in Fig. 3 were calculated from the respective functional relationships according to the GUM principle and are given with a coverage factor of $k=2$. The uncertainties of the diameters were estimated by repeated image evaluation. This results in a standard deviation of up to $u\left(d_{0}\right)=0.08$ pixel for cold images and up to $u(d(T))=0.15$ pixel for hot images, respectively. These maximum observed values were doubled to account for possible systematic effects and taken as worst case estimate for diameter uncertainty, independent of time. The temperature uncertainty was calculated as discussed in [29]. 


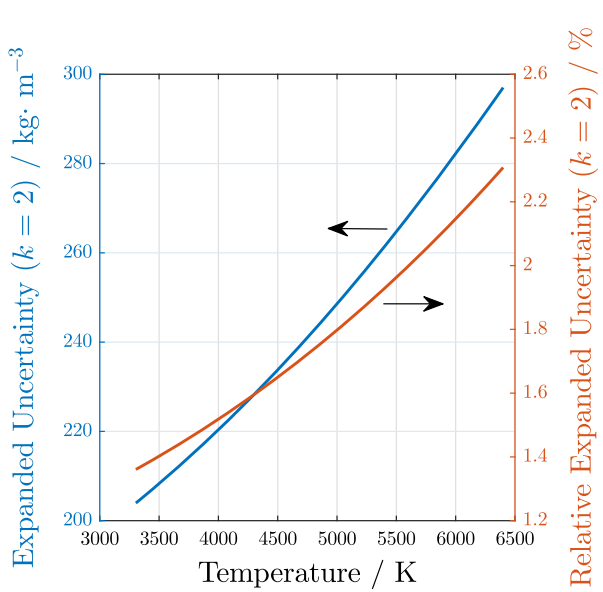

(a)

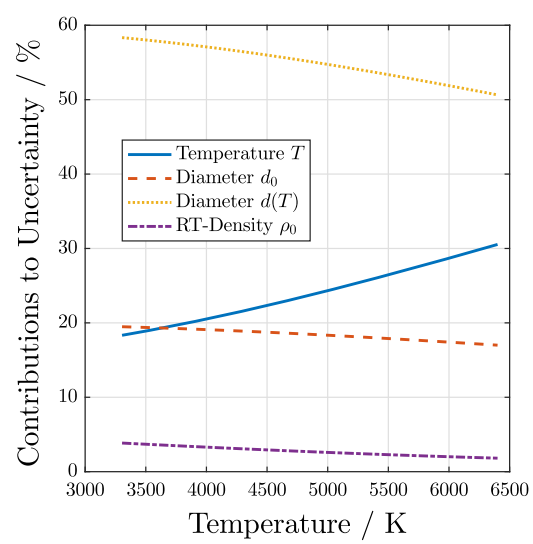

(b)

Fig. 5 Uncertainty of liquid-phase density-fit estimated according to GUM. (a) Expanded uncertainty and relative expanded uncertainty $(k=2)$ as a function of temperature. (b) Uncertainty budget as a function of temperature

In a second step, the uncertainties of the intercept $a$ and the slope $b$ of the linear density regression were calculated according to GUM, see [30], by including the individual $x$ - and $y$-uncertainties of the data points. The room temperature density uncertainty was adopted from [31], $u\left(\rho_{0}\right)=20 \mathrm{~kg} \cdot \mathrm{m}^{-3}$, and was combined with the uncertainty of the intercept after completing the evaluation described above.

Finally, the uncertainty of the fit at any fixed temperature $T$ was calculated according to GUM by employing the uncertainties of the fit coefficients and the temperature uncertainty to the fit equation. The absolute and relative expanded fit uncertainty $(k=2)$ thus obtained is depicted as a function of temperature in Fig. 5a. In addition, the percentual contributions of temperature $T$, room temperature diameter $d_{0}$, temperature-dependent diameter $d(T)$ and room temperature density $\rho_{0}$ to this uncertainty are shown in Fig. 5b. As can be seen, the temperature-dependent diameter $d(T)$ accounts for more than one half of the uncertainty over the entire liquid measuring range.

\subsection{Critical Point Data}

The uncertainty of the critical point $\left(T_{\mathrm{c}}, \rho_{\mathrm{c}}\right)$ was estimated from the GUM conform uncertainties of the intercept $a$ and the slope $b, b>0$, in the liquid-phase density regression. The critical point was calculated for the pairs $(a, b),(a+u(a), b-u(b))$ and $(a-u(a), b+u(b))$, where $u(x)$ denote the uncertainties of $x$ with $k=1$. The uncertainties of the critical point are then given as the doubled $(k=2)$ standard deviation of the mean value calculated from the three evaluations. Note that this reported uncertainty thus only represents the uncertainty range that originates from the uncertainty of the density regression coefficients. 


\section{Conclusion}

The density of tantalum was re-measured as a function of temperature over the liquid phase and into the super-heated region. The change in normal spectral emissivity over the measuring range was taken into account in order to minimize the uncertainty due to temperature measurement. The experimental data agree very well with previously published results and exhibit uncertainties of less than $2.3 \%$.

From the liquid-phase density behavior, the critical temperature and critical density were estimated and compared with theoretical results reported in the literature. The concordance is remarkably good.

Acknowledgements Open access funding provided by Graz University of Technology.

Open Access This article is distributed under the terms of the Creative Commons Attribution 4.0 International License (http://creativecommons.org/licenses/by/4.0/), which permits unrestricted use, distribution, and reproduction in any medium, provided you give appropriate credit to the original author(s) and the source, provide a link to the Creative Commons license, and indicate if changes were made.

\section{References}

1. W. Schröer, G. Pottlacher, High Temp. High Press. 43, 201 (2014)

2. M. Leitner, T. Leitner, A. Schmon, K. Aziz, G. Pottlacher, Metall. Mater. Trans. A 48, 3036 (2017)

3. A. Schmon, K. Aziz, M. Luckabauer, G. Pottlacher, Int. J. Thermophys. 36, 1618 (2015)

4. A. Schmon, K. Aziz, G. Pottlacher, Metall. Mater. Trans. A 46, 2674 (2015)

5. K. Boboridis, G. Pottlacher, H. Jäger, Int. J. Thermophys. 20, 1289 (1999)

6. E. Kaschnitz, G. Pottlacher, High pressure-high temperaturethermophysical measurements on liquid metals (Teubner, 1992). Teubner-Texte zur Physik 26, 139-144 (1992)

7. C. Cagran, T. Hüpf, B. Wilthan, G. Pottlacher, High Temp. High Press. 37, 205 (2008)

8. E. Kaschnitz, G. Pottlacher, H. Jäger, Int. J. Thermophys. 13, 699 (1992)

9. T. Hüpf, Density Determination of Liquid Metals. Ph.D. thesis, Graz, University of Technology (2010)

10. A. Schmon, Density Determination of Liquid Metals by Means of Containerless Techniques. Ph.D. thesis, Graz University of Technology (2016)

11. R. Bedford, G. Bonnier, H. Maas, F. Pavese, Metrologia 33, 133 (1996)

12. C. Cagran, C. Brunner, A. Seifter, G. Pottlacher, High Temp. High Press. 34, 669 (2002)

13. G. Pottlacher, A. Seifter, Int. J. Thermophys. 23, 1267 (2002)

14. G. Pottlacher, T. Hüpf, Thermal Conductivity 30/Thermal Expansion 18 (DEStech Publications, Inc., Lancaster, 2010), pp. 192-206

15. Y. Marcus, J. Chem. Thermodyn. 109, 11 (2016)

16. G.R. Gathers, Int. J. Thermophys. 4, 149 (1983)

17. A. Berthault, L. Arles, J. Matricon, Int. J. Thermophys. 7, 167 (1986)

18. H. Jäger, W. Neff, G. Pottlacher, Int. J. Thermophys. 13, 83 (1992)

19. P.F. Paradis, T. Ishikawa, S. Yoda, Appl. Phys. Lett. 83, 4047 (2003)

20. D.R. Lide (ed.), CRC Handbook of Chemistry and Physics, vol. 85 (CRC Press, Boca Raton, 2004)

21. J.W. Shaner, G.R. Gathers, C. Minichino, High Temp. High Press. 9, 331 (1977)

22. A. Likalter, Phys. A 311, 137 (2002)

23. A. Grosse, J. Inorg. Nucl. Chem. 22, 23 (1961)

24. S. Blairs, M.H. Abbasi, Acta Acust. Acust. 79, 64 (1993)

25. S. Blairs, M.H. Abbasi, J. Colloid Interface Sci. 304, 549 (2006)

26. V. Fortov, K. Khishchenko, P. Levashov, I. Lomonosov, Nucl. Instrum. Methods Phys. Res. Sect. A 415, 604 (1998)

27. H. Hess, H. Schneidenbach, Z. Metallkd. 87, 979 (1996) 
28. W.G. of the Joint Committee for Guides in Metrology (JCGM/WG 1). Evaluation of measurement data-guide to the expression of uncertainty in measurement (JCGM-Joint Committee for Guides in Metrology) (2008)

29. B. Wilthan, Verhalten des Emissionsgrades und thermophysikalische Daten von Legierungen bis in die flüssige Phase mit einer Unsicherheitsanalyse aller Messgrößen. Ph.D. thesis, Graz, University of Technology (2005)

30. M. Matus, Tech. Mess. 72, 584 (2005)

31. L.C. Ming, M.H. Manghnani, J. Appl. Phys. 49, 208 (1978) 